\title{
26 Research Square \\ Experimental analysis on the optimal excitation wavelength for fine-grained identification of refined oil pollutants on water surface based on laser- induced fluorescence
}

\author{
Ming Xie \\ Dalian Maritime University \\ Yunpeng Jia \\ Dalian Maritime University \\ Ying Li ( $\square$ yldmu@dlmu.edu.cn ) \\ Dalian Maritime University https://orcid.org/0000-0002-4318-591X \\ Xiaohua Cai \\ Dalian Maritime University \\ Kai Cao \\ Dalian Maritime University
}

\section{Research Article}

Keywords: Laser-induced fluorescence, fluorometric spectra analysis, oil spill, oil types classification, finegrained classification

Posted Date: October 19th, 2021

DOI: https://doi.org/10.21203/rs.3.rs-756586/v2

License: (c) (1) This work is licensed under a Creative Commons Attribution 4.0 International License.

Read Full License

Version of Record: A version of this preprint was published at Journal of Fluorescence on November 11th, 2021. See the published version at https://doi.org/10.1007/s10895-021-02849-3. 


\section{Abstract}

Laser-induced fluorescence (LIF) is an effective, all-weather oil spill identification method that has been widely applied for oil spill monitoring. However, the distinguishability on oil types is seldom considered while selecting excitation wavelength. This study is intended to find the optimal excitation wavelength for fine-grained classification of refined oil pollutants using LIF by comparing the distinguishability of fluorometric spectra under various excitation wavelengths on some typical types of refined-oil samples. The results show that the fluorometric spectra of oil samples significantly vary under different excitation wavelengths, and the four types of oil applied in this study are most likely to be distinguished under the excitation wavelengths of $395 \mathrm{~nm}$ and $420 \mathrm{~nm}$. This study is expected to improve the ability of oil types identification using LIF method without increasing time or other cost, and also provides theoretical basis for the development of portable LIF devices for oil spill identification.

\section{Introduction}

As the fast development of offshore oil exploitation and maritime transportation, oil spill events caused by leakages from platforms and collisions of ships frequently occurred and negatively affected maritime transportation and ocean environment (Fingas and Brown, 2013a; Alves et al., 2016). With the awareness of the destructive impacts of oil spills, the detection and prevention of oil spill events have become an important and interdisciplinary topic. Thanks to the international cooperation on the oil spill prevention and response, such as the International Convention on Oil Pollution Preparedness, Response, and Cooperation (OPRC), the number of large oil spill events has significantly decreased in the past few decades. However, medium and small oil spill events (from 7 to 700 ton) still occurred frequently and endangered ocean environment (ITOPE, 2021). Besides the collisions of ships, medium and small oil spill events could also be caused by load, unload, ballast, fuel charging, tank cleaning, and various maritime activities. Refined oil, such as diesel, gasoline, and lubricant, are commonly witnessed in medium and small oil spill events (Loh et al., 2021).

Oil types identification is a fine-grained classification problem that can help determine the source of leakage and the plan for responses. Chemical methods, such as chromatographic/mass spectroscopic analysis on oil samples (Texeira et al., 2014; Bayona et al., 2015), and "oil spill fingerprint" methods (Christensen and Tomasi, 2016; Liu et al., 2017; Boehmer- Christiansen, 2008) can provide accurate inferences on oil spill types. However, these methods usually require in situ sampling and large analytical equipment. Thus, they could be time-consuming, and usually not fit for medium and small oil spill events. It is necessary to develop a fast and accurate method for oil types identification in medium and small oil spill events.

Almost all types of petroleum hydrocarbons (PHCs) have unique fluorometric characteristics, which can be used for PHC identification on soil, sea water, ice, and other complicated background (Fingas and Brown, 2013b; Araújo et al., 2021). Therefore, laser-induced fluorescence (LIF) is an effective, all-weather oil spill identification method that has been widely applied for oil spill monitoring. Brown et al. (1996) 
designed an airborne laser fluorosensors and conducted some field tests. Hou et al. $(2017,2019,2021)$ conducted a series of studies on the fluorometric characteristics of aromatic hydrocarbons in different types of oil, and designed a port-based device for oil spill monitoring based on LIF. Although LIF could effectively identify the present of oil pollutants, it is difficult to provide fine-grained classification between various types of oil that have similar fluorometric spectra. In order to solve this problem, Chekalyuk and Hafez (2013) proposed an "Advanced Laser Fluorometry" (ALF) method by using laser with multiple excitation wavelengths. However, both LIF and ALF methods overlooked the selection of excitation wavelengths: they usually determine the excitation wavelength based on laser frequency doubling of the equipment or excitation efficiency of the material (Brown and Fingas, 2003). Distinguishability is seldom considered while selecting excitation wavelength. In order to have a comprehensive understanding on the characteristics of LIF, researchers combined the fluorometric spectra under different excitation wavelengths and formed excitation-emission matrix (EEM) (Baszanowska and Otremba). EEM introduces additional information in the dimension of excitation wavelength, and thus potentially improves the accuracy of oil types identification (Christensen et al., 2005). However, the measurements of EEM are also costly, time-consuming, and difficult to applied in real situation (especially for medium and small oil spill event) (Hou et al., 2017).

Considering the limitations in the previous works on LIF and EEM, this study is intended to compare the distinguishability of fluorometric spectra under various excitation wavelengths on some typical refine oil samples, and find the optimal excitation wavelength for oil types identification using LIF. By examining and analysing EEMs of different oil types, this study is expected to improve the ability of oil spill classification using LIF method without increasing time or other cost. Furthermore, this study is expected to provide theoretical basis for the development of portable LIF devices for oil spill identification.

\section{Methodology}

\subsection{Equipment and material}

In the experiment, fluorescence is excited using a laser xenon lamp and collected using a hyperspectral sensor. An adjustable xenon lamp is used as the light source for excitation in order to collect the fluorometric spectra at different excitation wavelengths. The model of the laser xenon lamp used in the experiment is TLS-300XR (Fig. 1a), which is produced by Oriel Instruments, Newport Corporation, America. The tunable spectral range of the laser xenon lamp is from $300 \mathrm{~nm}$ to $1800 \mathrm{~nm}$, and its spectral resolution is $5 \mathrm{~nm}$. Only the ultraviolet part that is strong enough to excite fluorescence is used for the purpose of the study.

Intensity of fluorescence is then measured using a portable hyperspectral sensor: Analytical Spectral Devices (ASD) FieldSpec3 (Fig. 1b). The response spectral range of the device is $350-2500 \mathrm{~nm}$. The spectral resolution is $3 \mathrm{~nm}$ at $700 \mathrm{~nm}$ and $10 \mathrm{~nm}$ at $1400 / 2000 \mathrm{~nm}$, and the sampling interval is $1.4 \mathrm{~nm}$ from $350 \mathrm{~nm}$ to $1050 \mathrm{~nm}$, and $2 \mathrm{~nm}$ from $1000 \mathrm{~nm}$ to $2500 \mathrm{~nm}$. 
Sea water and various types of oil samples were prepared for the purpose of the experiment. Seawater samples were collected in the port of Lingshui, China, in the northern Yellow Sea. Diesel, gasoline, and two types of lubricant are selected as the objects of identification in this study: (1) 0\# diesel, which solidifies at $0^{\circ} \mathrm{C}$ and has a density of $830 \mathrm{~kg} / \mathrm{m}^{3}$. It is commonly used as ship fuel; (2) 92\# gasoline, which contains $92 \%$ isooctane and $8 \%$ heptane, and has a density of $730 \mathrm{~kg} / \mathrm{m}^{3}$. It is commonly used as fuel for small ships; (3) Mobile $0 W 40$, of which the density is $850 \mathrm{~kg} / \mathrm{m}^{3}$. It is commonly used as lubricant for gasoline engine; (4) DAZIRAN CF-4, of which the density is $880 \mathrm{~kg} / \mathrm{m}^{3}$. It is a commonly used as lubricant for diesel engine. Fig. 2 shows the appearances of these four types of oil samples.

Multiple measurements on the fluorometric spectra of oil samples are conducted under different oil thicknesses and excitation wavelengths in order to form the training dataset for the classifier, and also test the robustness of the classifier (whether the classifier is able to distinguish oil samples at different thicknesses). $1 \mathrm{ml}, 2 \mathrm{ml}$, and $4 \mathrm{ml}$ of oil samples are collected using pipette and dropped into glass bottles with seawater. The bottles with oil samples are placed statically until stable oil films are formed. Since the oil samples used in this study are refined oil and relatively easy to spread on water surface, they did not need to be warmed before use, and usually took less than 1 minute to form stable oil films. However, since some of the oil samples can easily volatilize, the bottles need to be covered with frosted caps. Additionally, a bottle with only sea water is prepared to measure the background fluorescence.

Oil thickness can be estimated using the diameter of the glass bottle and the volume of the oil sample. The glass bottles used in the experiments are $40 \mathrm{~mm}$ in diameter and $25 \mathrm{~mm}$ in height. Thus, the oil thicknesses of $1 \mathrm{ml}, 2 \mathrm{ml}$, and $4 \mathrm{ml}$ oil samples are about $0.8 \mathrm{~mm}, 1.6 \mathrm{~mm}, 3.2 \mathrm{~mm}$, respectively. It should be noted that although the oil thickness is recorded in the experiment, it is not fed to the classifier in order to test whether the model is able to recognize the types of oil without pre-knowledge about oil thickness. 1000 fluorometric spectra are collected for each type of oil under each set of oil thickness and excitation wavelengths. Thus, there are totally 15,000 fluorometric spectra collected to form the training and testing data under each set of excitation wavelengths. $80 \%$ of the collected EEMs are used as training dataset and $20 \%$ of those are used as testing data.

\subsection{Experiment design}

Fluorometric spectra under different excitation wavelengths are collected in experiments as the training and testing dataset of the classifier. The experiment is conducted under dark laboratory condition in order to prevent the inferences of ambient light on LIF of the oil samples. The range of excitation wavelength $\left(\lambda_{e x}\right)$ from laser xenon lamp is set at $355-455 \mathrm{~nm}$, and the step length is set at $5 \mathrm{~nm}$. Therefore, the fluorometric spectra are collected under 21 different excitation wavelengths. The classifier makes prediction using the fluorometric spectra collected under each of the 21 bands, and the optimal excitation wavelength can be determined by examining the accuracies of classification results under these 21 bands. 
The range of emission wavelength $\left(\lambda_{e m}\right)$ is set at $370-700 \mathrm{~nm}$ at the spectra resolution of $1 \mathrm{~nm}$. Thus, there are 351 bands in each spectrum. In order to avoid the influence from Rayleigh scattering, the emission wavelengths lag behind the excitation wavelengths by $15 \mathrm{~nm}$. A schematic diagram of the experiment is shown in Fig. 3a, and a photograph of the experiment setting is shown in Fig. 3b.

1000 fluorometric spectra are collected for each type of oil under each set of oil thickness and excitation wavelengths. Thus, there are totally 15,000 fluorometric spectra collected to form the training and testing data under each set of excitation wavelengths. $80 \%$ of the collected fluorometric spectra are used as training dataset and $20 \%$ of those are used as testing data.

\subsection{Implementations of classifier}

After the fluorometric spectra are collected for the four types of oil at different thicknesses and under different excitation wavelengths. 21 identification experiments are conducted using the fluorometric spectra collected under each set of excitation wavelengths. The accuracies of the experiments are evaluated and compared in order to find the optimal excitation wavelength for oil types classification.

Because the types of oil are labelled when taking fluorescence measurements, regular supervised learning methods that provide more accurate classification with smaller number of calculations are preferable for the oil types identification task. Bagged random forest (RF) is selected as the classifier in this study. RF is a classical method of supervised machine learning that consists of a number of tree-like classification units and a majority vote (Breiman, 2001). Each classification unit makes prediction based on its own decision tree. The votes from all classification units are collected and the prediction with largest number of votes is considered as the final output (Liaw and Wiener, 2002). RF is able to handle high-dimensional data effectively, and has been applied to spectral analysis and classification (Belgiu and Dragut, 2016).

In this study, the whole spectrum that consists of 351 bands is considered as the input data. The size of output is 4 , which presents for the 4 categories of oil used in the experiments. The bagged RF model consists of 200 tree-like classification units. The working flow of RF applied in this study is shown as Fig. 4. The model is constructed using Statistical and Machine Learning Toolbox in MATLAB R2019a, and trained on a computer of $2.5 \mathrm{GHz}$ Intel i5-7300HQ CPU and $8.0 \mathrm{~GB}$ of RAM.

\section{Results}

\subsection{Laser induced fluorometric spectra}

The collected fluorometric spectra of oil samples are corrected with those of the sea water sample in order to eliminate the background fluorescence. Two examples of the processed fluorometric spectra collected under excitation wavelengths of $385 \mathrm{~nm}$ and $420 \mathrm{~nm}$ are shown as Fig. 5 .

The fluorometric spectra at all 21 different excitation wavelengths can be combined and form EEM in order to provide better visualization and conduct more comprehensive analysis on the fluorometric spectra. EEMs generated using the fluorometric spectra of four types of oil are shown as Fig. 6. As shown 
in the EEMs, obviously, there are significant differences in the fluorometric spectra collected under different excitation wavelengths. Therefore, it is necessary to examine the distinguishability of LIF method under different wavelengths.

\subsection{Oil types identification at different excitation wavelength}

As mentioned above in the methodology part, 21 classification experiments are conducted using the fluorometric spectra under each of the excitation wavelengths, and the accuracies of all experiments are compared in order to determine the optimal wavelength. The accuracies of identification results are quantitatively evaluated through producers' accuracies (PA), users' accuracies (UA), and overall accuracies (OA). PA, UA, OA for the four types of oil under all excitation wavelengths are plotted in Fig. 7. Additionally, the general accuracy of the model, which is defined as the ratio between the number of all correct predictions and that of all test data, are plotted in Fig. 8.

\section{Discussion}

\subsection{Interpretation of fluorometric spectra}

According to the results, the fluorescence intensities of diesel and diesel engine oil are relatively low, and have similar pattern under the excitation wavelengths of 385 and $420 \mathrm{~nm}$. The fluorescence intensities of 92\# gasoline and gasoline engine oil are relatively higher. 92\# gasoline has similar peak of fluorescence at about $450 \mathrm{~nm}$ under these two excitation wavelengths; while that for 0W40 shifts from $450 \mathrm{~nm}$ to 520 $\mathrm{nm}$ under these two excitation wavelengths. The shapes of fluorometric spectra for diesel and diesel engine oil are visually identical under these two excitation wavelengths. The peaks of fluorescence for these two types of oil shift from $440 \mathrm{~nm}$ to about $500 \mathrm{~nm}$ under the two excitation wavelengths, while the peaks are not as significant as those of gasoline and gasoline lubricant.

According to the generated EEMs in Fig. 6, these four different types of oil have similar peak of fluorescence at about $450 \mathrm{~nm}$ under the excitation wavelength of $380 \mathrm{~nm}$. 0W40 have an additional peak of fluorescence at about $550 \mathrm{~nm}$ under the excitation wavelength of $445 \mathrm{~nm}$. These results generally conform with the observations of LIF in previous studies (Brown and Fingas, 2003; Christensen et al., 2005; Baszanowska and Otremba, 2019; Loh et al., 2021).

\subsection{Optimal excitation wavelength for oil types identification}

According to the $\mathrm{OA}$ of the classification results for the four types oil under all excitation wavelengths (Fig. 7c), the classifier has low identification accuracies under the excitation wavelengths from $365 \mathrm{~nm}$ to $385 \mathrm{~nm}$. Diesel and diesel engine oil are also difficult to be identified over $440 \mathrm{~nm}$, while gasoline and gasoline engine oil also have low identification accuracies from $400 \mathrm{~nm}$ to $410 \mathrm{~nm}$. 
Referring to the general accuracy graph (Fig. 8), the model is generally not able to provide very accurate classification results in the spectral range of excitation laser described above. Combining the OA for the identification results of all four types of oil, the model is able to achieve identification results with the highest accuracy under the excitation wavelengths $395 \mathrm{~nm}$ and $420 \mathrm{~nm}$. This result indicates that the four types of oil applied in this experiment are most distinguishable under these two excitation wavelengths.

\section{Conclusion}

The selection of the optimal excitation wavelength for fine-grained classification of refined oil pollutants using LIF is studied through experimental analysis in this paper. Comparing the accuracies of the identification results using the fluorometric spectra collected under different excitation wavelengths, it seems that the four types of oil are most likely to be distinguished under the excitation wavelengths of $395 \mathrm{~nm}$ and $420 \mathrm{~nm}$. This study provides a guidance to the choices of excitation laser for oil types identification using LIF method. In the future study, the authors' team will consider include more types of oil pollutants (and even the mixture of different types of oil) in the experiment in order to establish an optimal excitation wavelength for distinguishing between broader types of oil pollutants.

\section{Declarations}

Ethics approval and consent to participate: Not applicable.

Consent for publication: Not applicable.

Data availability statement: The data and codes that support the findings of this study are publicly available online at https://github.com/349898680/Dr-Xiecode/tree/main/CNN\%20model\%20for\%20EEM\%20of\%20oil.

Competing interests: The authors declare no conflicts of interest.

Funding. This research was funded in part by China National Key R\&D Program [grant number 2020YFE0201500], Liaoning Revitalization Talents Program [grant number XLYC2001002] and in part by Postdoctoral Science Foundation of China [grant number 2020M670730].

Authors' contribution. MX designed the methodology, constructed the identification model, prepared the original draft, and completed the reviewing and editing of the paper. YJ conducted the experiment, processed the data, and participated in the preparation of original draft. YL proposed the concept, provided supervision and completed the reviewing and editing of the paper. XC participated in the experiment and data processing, as well as results investigation. $\mathrm{KC}$ coded the software, investigates and validated the classification results. All authors read and approved the final manuscript. 
Acknowledgements. The authors would like to thank Dr. Zhenduo Zhang and Dr. Yu Liu from Dalian Maritime University for providing some of the oil samples. The authors would also like to thank Zhanjun Ma, Wenbo Zheng, and Qinglai Yu from Dalian Maritime University for their help in preparing the training dataset.

\section{References}

Alves TM, Kokinou E, Zodiatis G, Radhakrishnan H, Panagiotakis C, Lardner R (2016) Multidisciplinary oil spill modeling to protect coastal communities and the environment of the Eastern Mediterranean Sea. Sci Rep 6(1):1. https://doi.org/10.1038/srep36882.

Araújo KC, Barreto MC, Siqueira AS, Freitas ACP, Oliveira LG, Eugênia M, Bastos PA., Rocha MEP, Silva LA, Fragoso WD (2021) Oil spill in northeastern Brazil: Application of fluorescence spectroscopy and PARAFAC in the analysis of oil-related compounds. Chemosphere 267:129154. https://doi.org/10.1016/j.chemosphere.2020.129154.

Baszanowska E, Otremba Z (2019) Detecting the presence of different types of oil in seawater using a fluorometric index. Sensors 19(17):3774. https://doi.org/10.3390/s19173774.

Bayona JM, Domínguez C, Albaigés J (2015) Analytical developments for oil spill fingerprinting. Trends Environ Anal Chem 5:26-34. https://doi.org/10.1016/j.teac.2015.01.004.

Belgiu M, Dragut L (2016) Random forest in remote sensing: A review of applications and future direction. ISPRS Int J Photogramm Remote Sens 114(3):24-31. https://doi.org/10.1016/j.isprsjprs.2016.01.011.

Boehmer-Christiansen S (2008) Estimates of oil entering the marine environment from sea-based activities, Reports and studies No. 75. Energy Environ 19(5):765-765. https://doi.org/10.1260/095830508784815955.

Breiman L (2001) Random forests. Mach Learn 45(1):5-31. https://doi.org/10.1023/A:1010933404324.

Brown CE, Fingas M (2003) Review of the development of laser fluorosensors for oil spill application. Mar Pollut Bull 47:477-484. https://doi.org/10.1016/S0025-326X(03)00213-3.

Brown CE, Nelson RD, Fingas F, Mullin JV (1996) Laser fluorosensors overflights of Santa Barbara oil seeps. Spill Sci Techno Bull 3(4):227-230. https://doi.org/10.1016/S1353-2561(97)00018-2.

Chekalyuk A, Hafez M (2013) Next generation Advanced Laser Fluorometry (ALF) for characterization of natural aquatic environments: new instruments. Opt Express 21(12):14181. https://doi.org/10.1364/OE.21.014181.

Christensen JH, Hansen AB, Mortensen J, Andersen 0 (2005) Characterization and matching of oil samples using fluorescence spectroscopy and parallel factor analysis. Anal Chem 77(7):2210-2217. https://doi.org/10.1021/ac048213k. 
Christensen JH, Tomasi G (2016) A multivariate approach to oil hydrocarbon fingerprinting and spill source identification. In: Stout SA, Wang Z (eds) Standard Handbook Oil Spill Environmental Forensics, 2nd edn, Elsevier, pp. 747-788. https://doi.org/10.1016/B978-0-12-803832-1.00016-7.

Fingas M, Brown CE (2013a) Oil spill remote sensing. In: Orcutt J (ed) Earth System Monitoring: Selected Entries from the Encyclopedia of Sustainability Science and Technology. Springer, pp. 337-388. https://doi.org/10.1007/978-1-4614-5684-1_15.

Fingas, M., Brown, CE (2013b) Detection of oil in ice and snow. J. Mar. Sci. Eng 1(1):10-20. https://doi.org/10.3390/jmse1010010.

Hou Y, Li Y, Liu B, Liu Y, Tong W (2017) Design and implementation of a coastal-mounted sensor for oil film detection on seawater. Sensors 18(1):70. https://doi.org/10.3390/s18010070.

Hou Y, Li Y, Liu Y, Li G, Zhang Z (2019) Effects of polycyclic aromatic hydrocarbons on the UV-induced fluorescence spectra of crude oil films on the sea surface. Mar Pollut Bull 146:977-984. https://doi.org/10.1016/j.marpolbul.2019.07.058.

Hou Y, Li Y, Li G, Xu M, Jia Y (2021) Species identification and effects of aromatic hydrocarbons on the fluorescence spectra of different oil samples in Seawater 2021:6677219. J Spectrosc https://doi.org/10.1155/2021/6677219.

ITOPE, 2021. Oil tanker spill statistics 2020. www.itopf.org/knowledge-resources/datastatistics/statistics/. Accessed 1 July 2021.

Liaw A, Wiener M (2002) Classification and Regression by RandomForest. R News 2/3:18-22.

Liu Y, Xu J, Chen W, Li Y (2017) Effects of short-term weathering on the stable carbon isotope compositions of crude oils and fuel oils. Mar Pollut Bull 119(1):238-244.

https://doi.org/10.1016/j.marpolbul.2017.04.003.

Loh A, Ha SY, Kim D, Lee J, Baek K, Yim UH (2021) Development of a portable oil type classifier using laser-induced fluorescence spectrometer coupled with chemometrics. J Hazard Mater 416:125723. https://doi.org/10.1016/j.jhazmat.2021.125723.

Texeira CC, Santos Siqueira CY, Aquino Neto FR, Miranda FP, Cerqueira JR, Vasconcelos AO, Landau L, Herrera M, Bannermaman K (2014) Source identification of sea surface oil with geochemical data in Cantarell, Mexico. Microchem J 117:202-213. https://doi.org/10.1016/j.microc.2014.06.025.

\section{Figures}




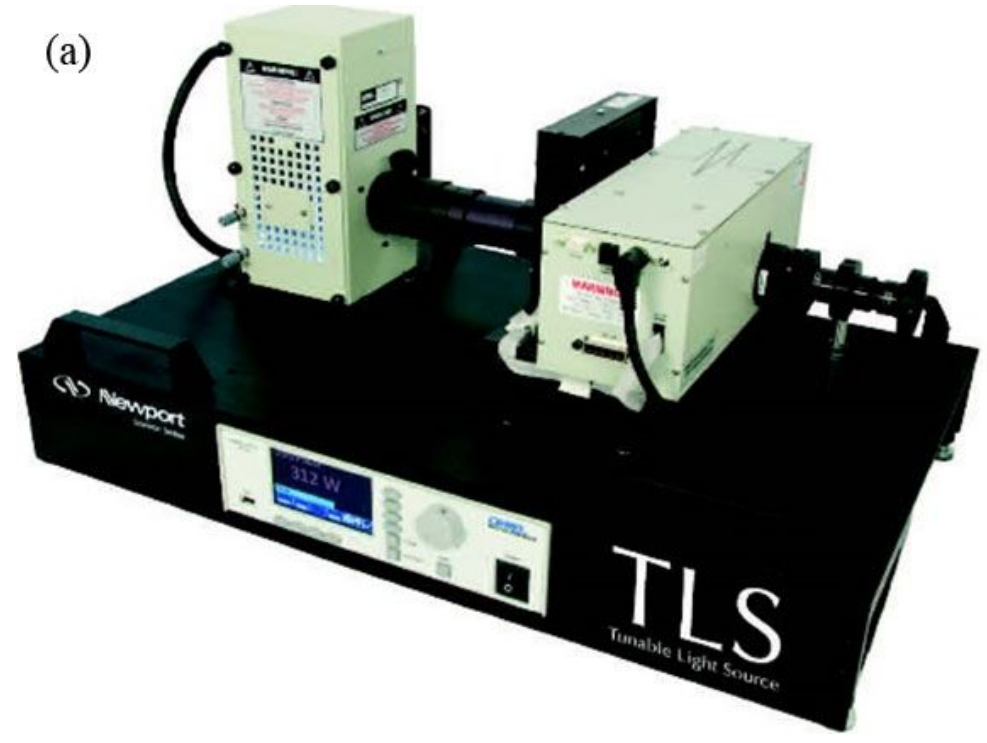

(b)

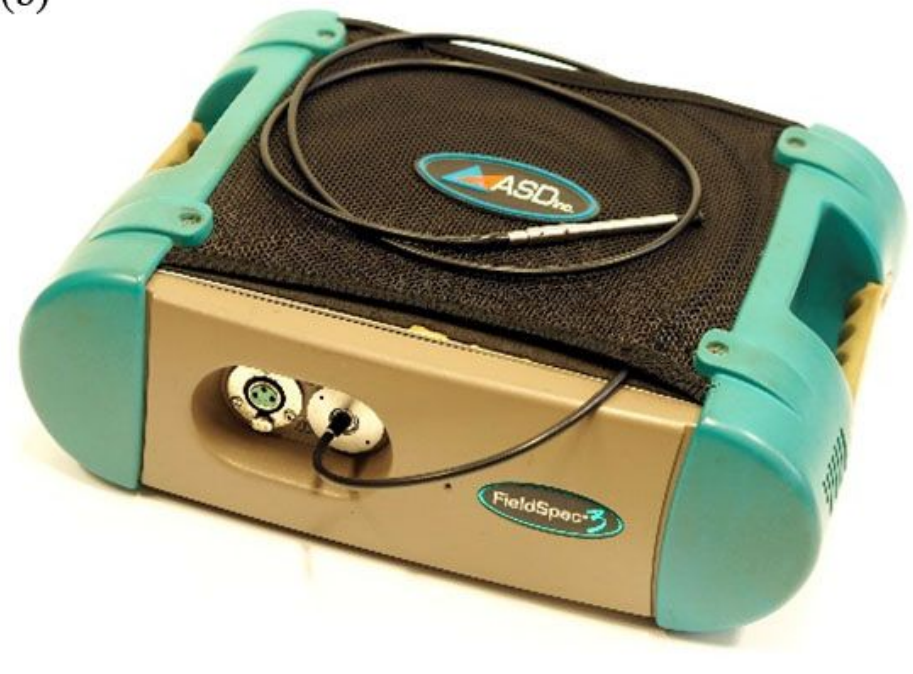

Figure 1

Photographs of the equipment that are used to collect fluorometric spectra: (a) TLS-300XR laser xenon lamp; (b) Analytical Spectral Devices (ASD) FieldSpec3 hyperspectral sensor.

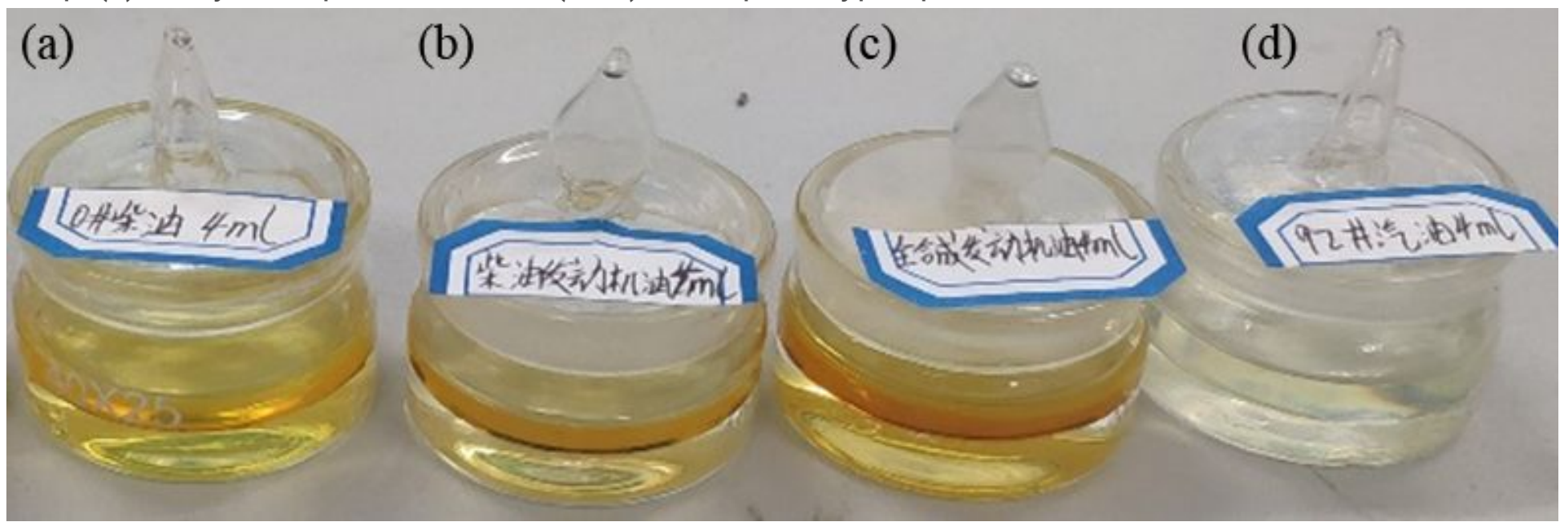

Figure 2

Photograph of the oil samples: (a) 0\# diesel; (b) CF-4; (c) OW40; (d) 92\# gasoline. 
(a)

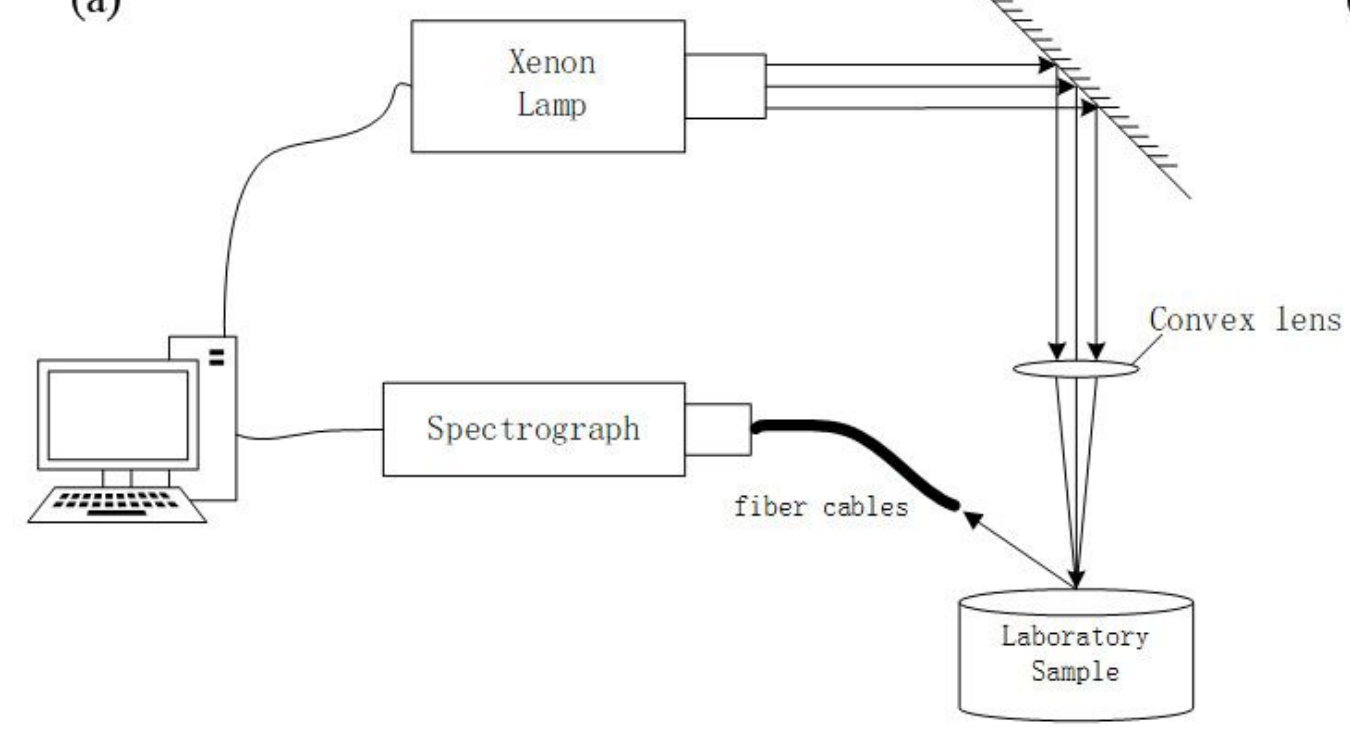

(b)

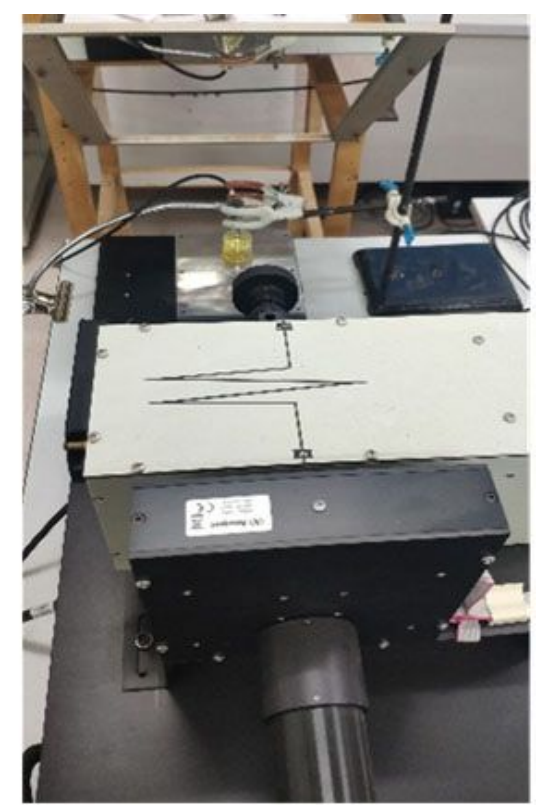

\section{Figure 3}

Experiment setting details: (a) schematic diagram; (b) photograph. 


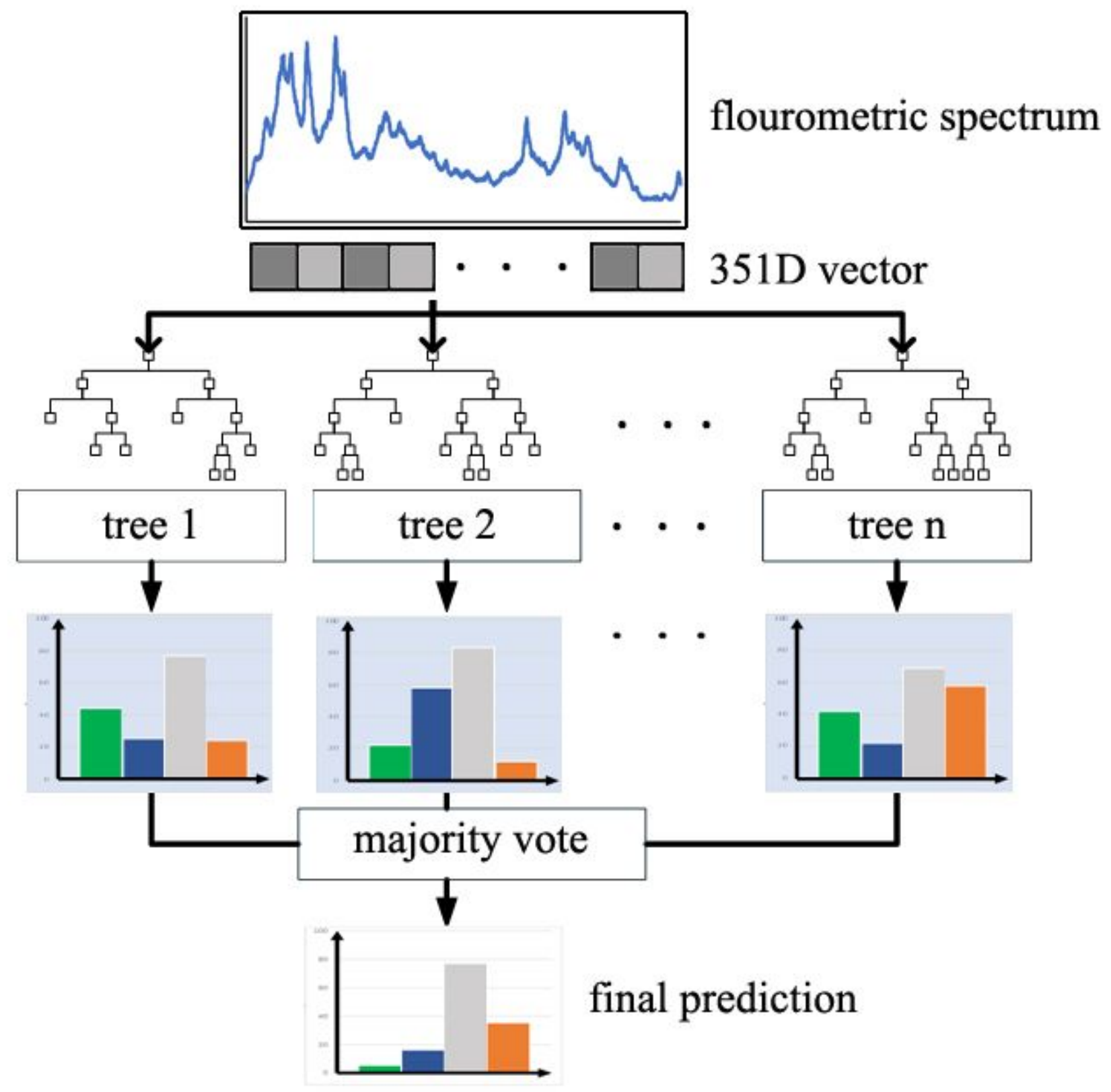

Figure 4

Overall structure of the bagged random forest $(\mathrm{RF})$ model applied in this study. 
(a)

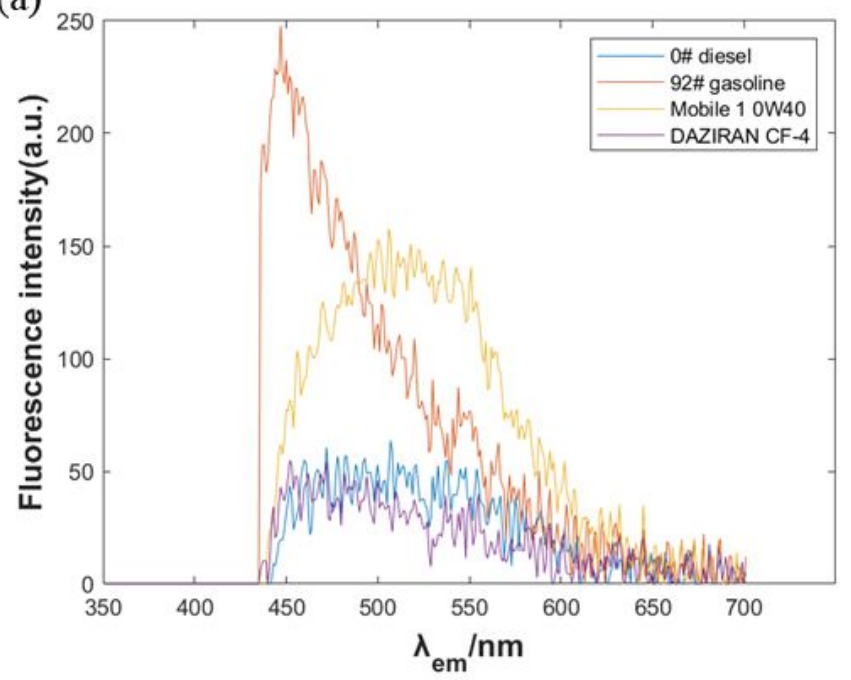

(b)

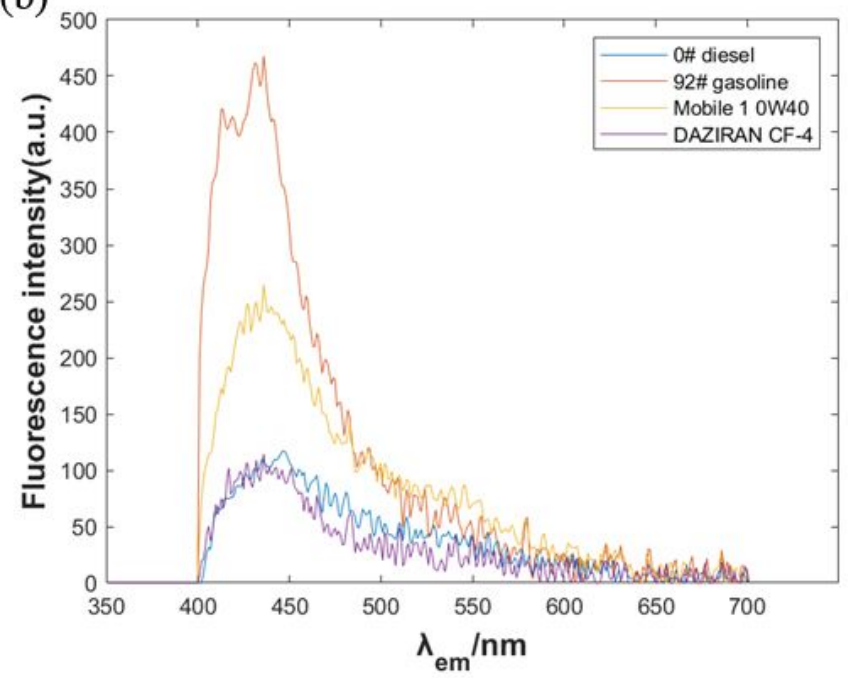

Figure 5

Fluorometric spectra under different excitation wavelength: (a) $385 \mathrm{~nm}$; (b) $420 \mathrm{~nm}$. 


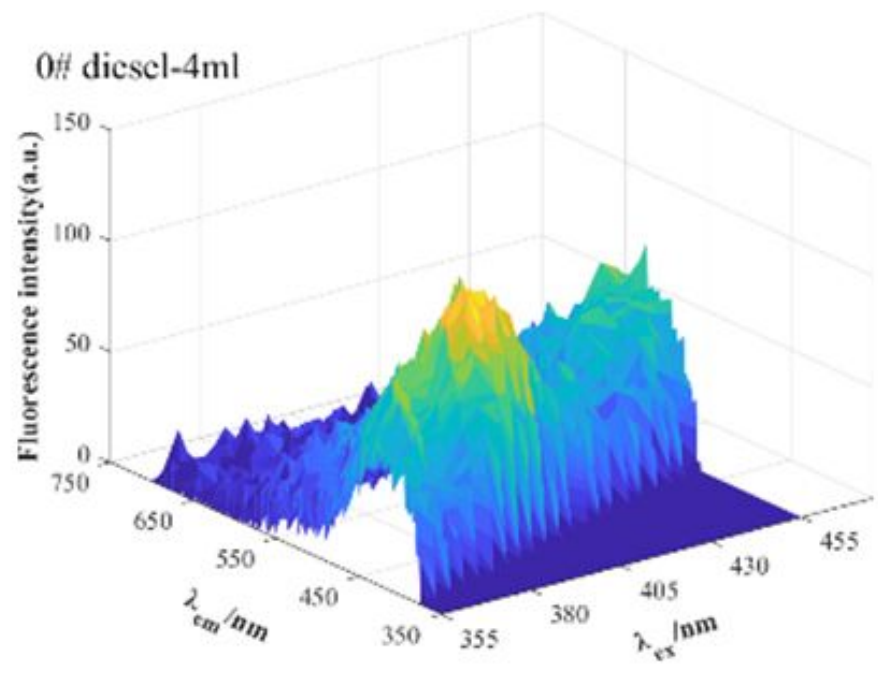

(a)

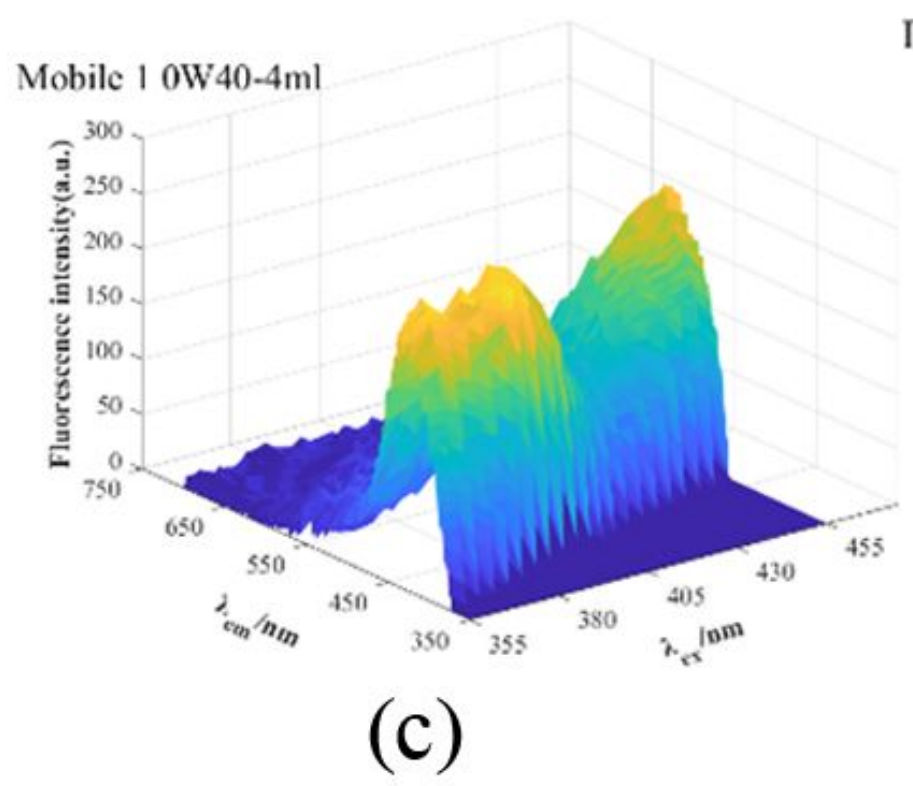

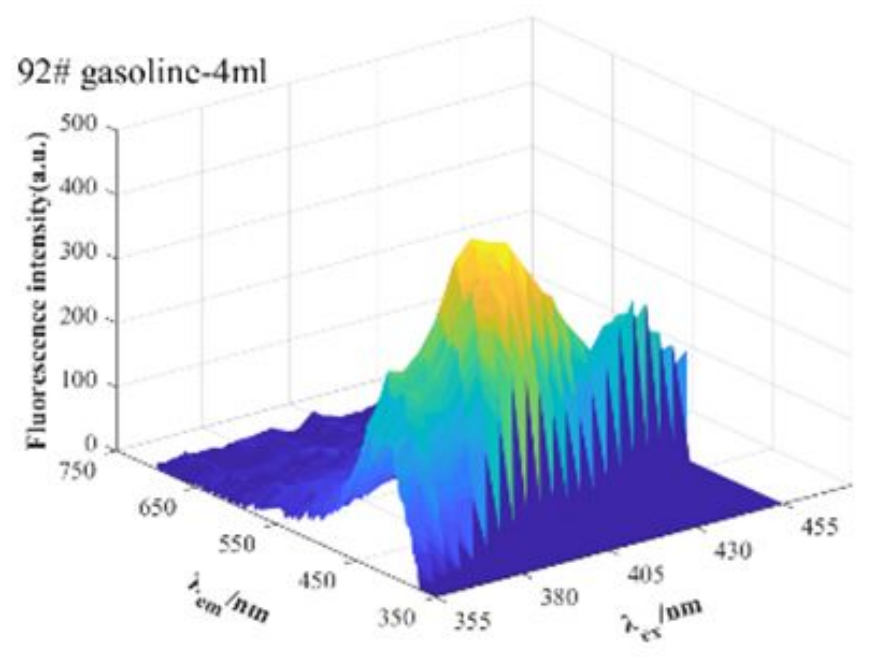

(b)

DAZIRAN CF-4-4ml

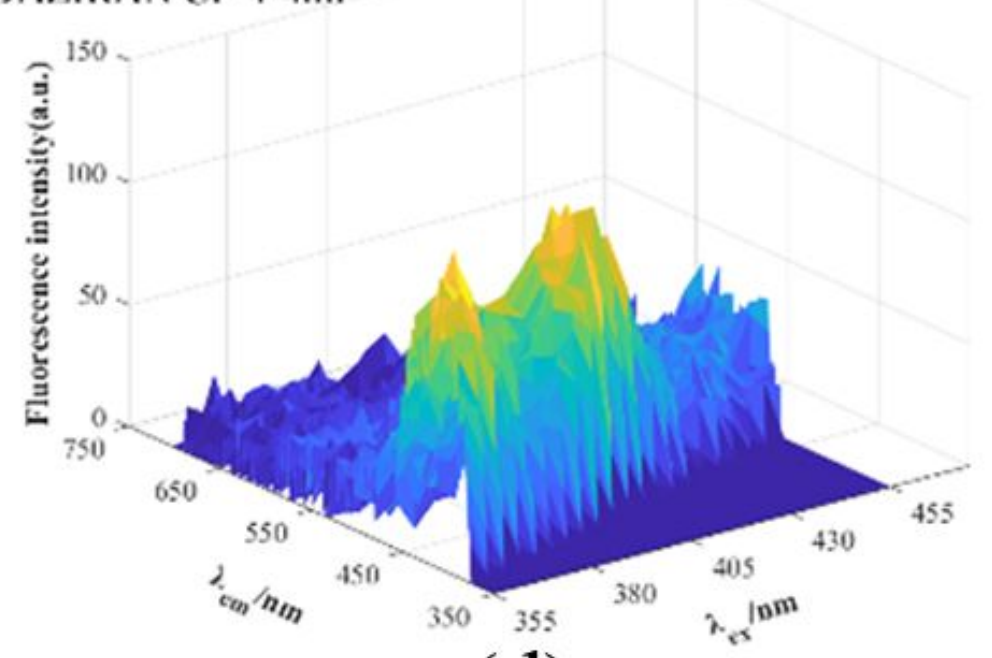

(d)

Figure 6

EEMs of four different types of oil used in the experiment: (a) 0\# diesel; (b) 92\# gasoline; (c) 0W40; (d) CF-4. 

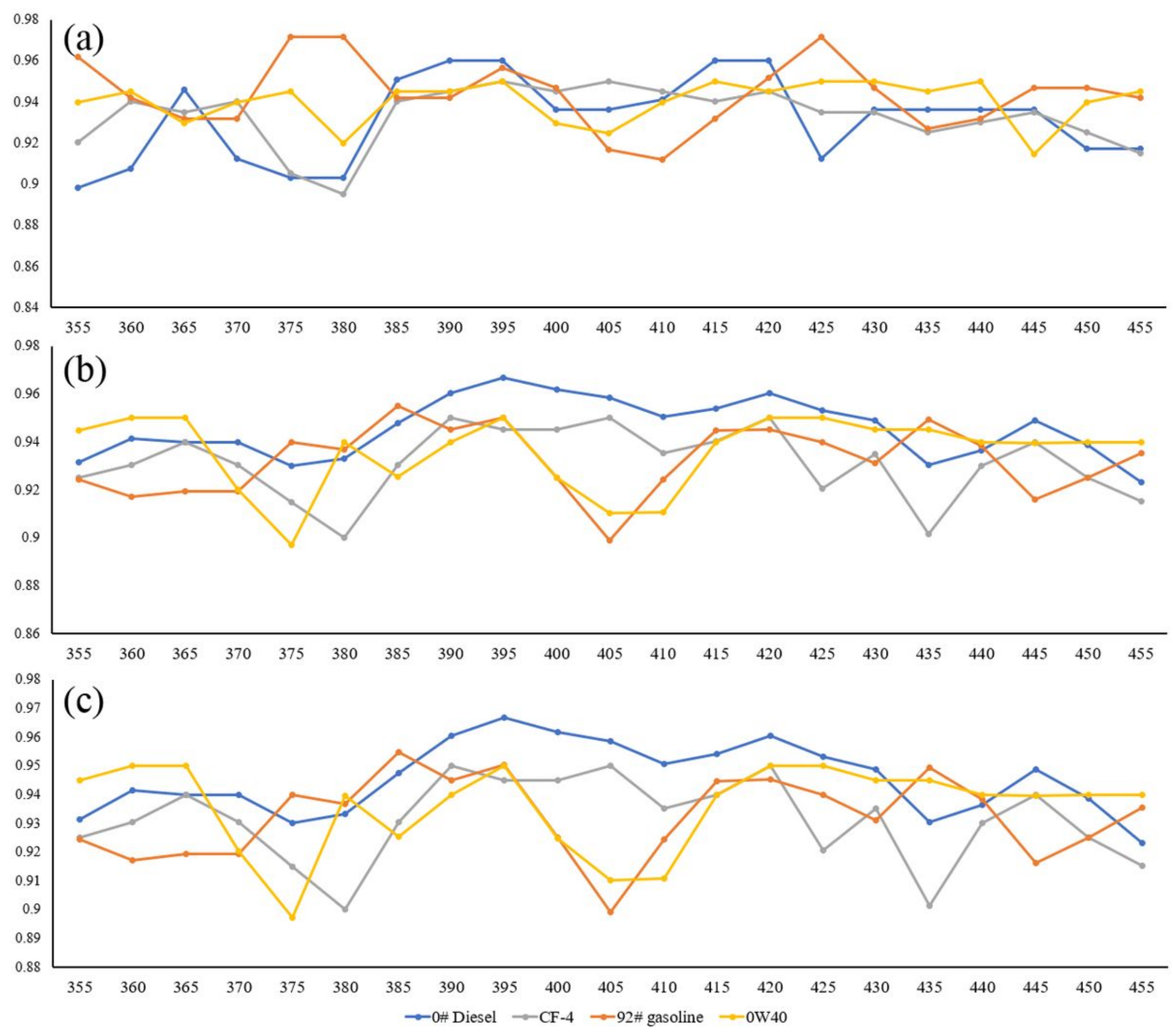

Figure 7

Accuracies of the identification results on four types of oil under different excitation wavelengths: (a) users' accuracy; (b) producers' accuracy; (c) overall accuracy 


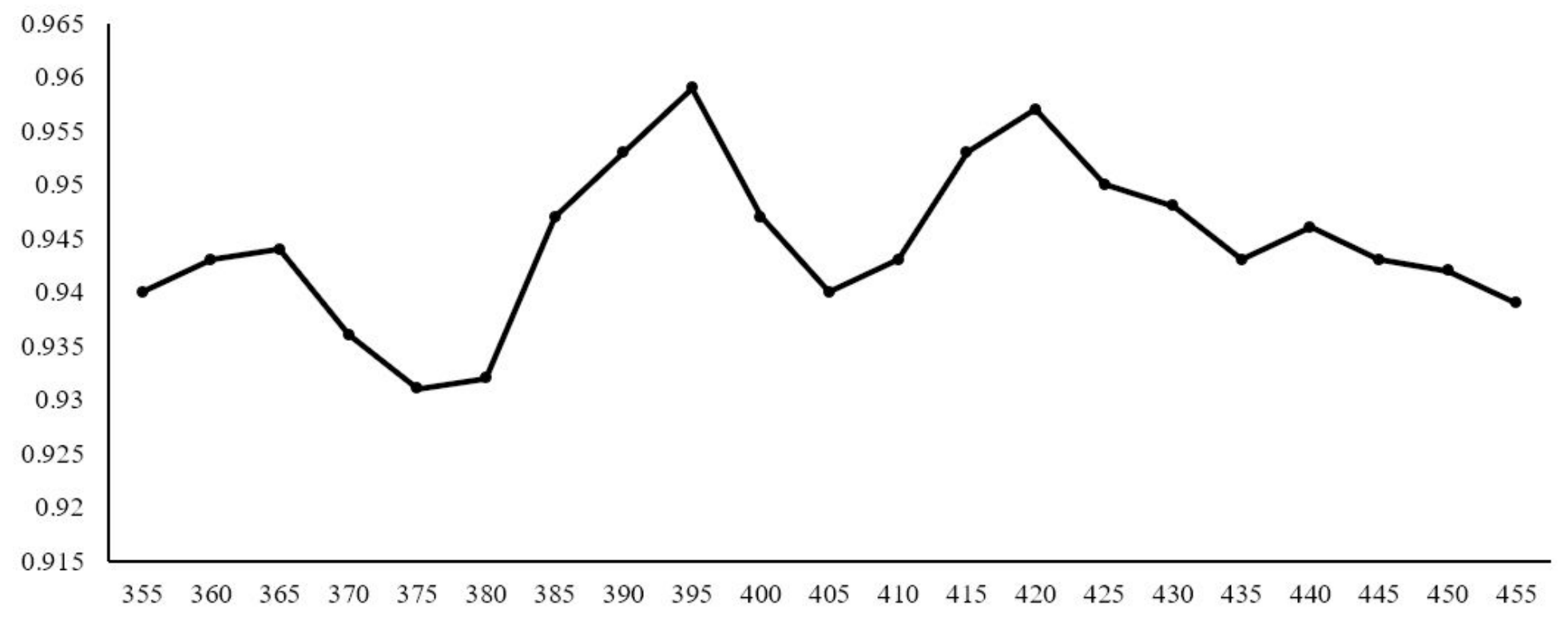

Figure 8

Classifier's general accuracies of the identification results under different excitation wavelengths. 